\title{
Photochimie supramoléculaire et complexes de coordination
}

\author{
E. Amouyal
}

\author{
Laboratoire de Chimie Physique, UMR 8000 du CNRS, Université Paris-Sud, bâtiment 350, \\ 91405 Orsay, France
}

\begin{abstract}
Résumé. La photophysique et la photochimie de complexes de coordination de métaux de transition a connu un essor considérable au cours de ces dernières années. D'une part, les complexes de coordination peuvent servir d'éléments de base dans l'élaboration de systèmes moléculaires et supramoléculaires; d'autre part, la lumière -outre son rôle de sonde et de caractérisation des molécules- peut être utilisée pour déclencher des processus spécifiques à ces systèmes. En particulier, le contrôle des processus de transfert d'électron photoinduits à l'échelle moléculaire constitue un défi important dans plusieurs domaines de recherche qui vont de la photosynthèse artificielle à l'électronique moléculaire. Dans ce cadre, nous présentons quelques exemples d'études photophysiques de molécules et de systèmes supramoléculaires à base de complexes polypyridiniques de ruthénium et d'osmium pour illustrer l'effet de l'organisation moléculaire sur les processus de transfert d'électron intramoléculaires.
\end{abstract}

\section{INTRODUCTION}

Un système supramoléculaire résulte de l'association d'au moins deux entités moléculaires (ions, molécules) liées entre elles par des forces intermoléculaires généralement faibles (liaisons hydrogène, forces de Van der Waals ou électrostatiques) [1]. Cette définition peut être étendue aux systèmes mettant en jeu des liaisons covalentes entre les différentes entités, à condition que chaque sous-unité moléculaire ait des propriétés intrinsèques bien définies et que le système résultant de cette association covalente présente de nouvelles propriétés que chacune des composantes ne possède pas. En d'autres termes, les propriétés des composantes de l'édifice supramoléculaire doivent être peu perturbées par rapport à celles de la molécule isolée correspondante [2]. De tels édifices sont aussi appelés diade, triade ou plus généralement polyade selon qu'ils comportent deux, trois ou plusieurs composantes.

L'interaction de la lumière avec les systèmes supramoléculaires est particulièrement intéressante car elle conduit à une très grande diversité de phénomènes. Elle constitue, par définition, le domaine d'étude de la photochimie supramoléculaire. En photochimie supramoléculaire, l'une des entités au moins est sensible à la lumière. Ainsi, on espère que l'organisation supramoléculaire va perturber les propriétés photophysiques et photochimiques des composantes pour donner lieu à des nouvelles propriétés, voire à de nouvelles fonctions spécifiques de l'édifice supramoléculaire. La lumière, en effet, peut avoir plusieurs rôles importants. Elle peut, en premier lieu, servir à caractériser et à sonder les systèmes supramoléculaires, en particulier en révélant les interactions entre les diverses composantes. Elle permet donc de mieux appréhender les relations entre la structure supramoléculaire et les propriétés physico-chimiques des molécules constitutives. La lumière permet également d'agir sélectivement sur un élément pour déclencher la fonction désirée, transformant ainsi le système supramoléculaire en un dispositif moléculaire photochimique. Enfin, la lumière peut être utilisée comme source d'énergie pour initier dans de tels dispositifs des réactions conduisant au transfert, à la conversion ou au stockage de cette énergie.

Les complexes de coordination de métaux de transition sont des éléments de choix pour concevoir et construire des édifices supramoléculaires photosensibles. En effet, leurs propriétés peuvent être modulées et ajustées par le choix judicieux du métal ou du ligand organique. Ce qui permet de contrôler les processus photoinduits nécessaires à l'obtention des fonctions désirées. Parmi ces processus, le transfert d'électron est un des plus importants en chimie et en biologie, en raison de son implication dans les phénomènes naturels (tels que la photosynthèse) et des perspectives d'applications dans divers domaines, de la conversion de l'énergie solaire à l'électronique moléculaire. Pour en illustrer l'intérêt, nous présentons quelques exemples 
de molécules et systèmes supramoléculaires, en examinant divers processus de transfert électronique intramoléculaire induits par la lumière.

\section{LOCALISATION ELECTRONIQUE}

L'acte photochimique primaire nécessite l'absorption d'un photon par les molécules ou les complexes de coordination. Dans ces derniers, la coordination entre le métal et le ligand organique se traduit par l'apparition d'une nouvelle bande d'absorption à caractère de transfert de charge, le plus souvent dans le domaine visible. L'excitation par la lumière va promouvoir un électron de l'état fondamental (état au repos) du complexe à son état excité le plus bas (état réactif). D'où l'intérêt - fondamental et appliqué - de mieux comprendre comment l'énergie d'excitation est distribuée dans cet état excité, en d'autres termes, de déterminer si l'électron promu est délocalisé sur l'ensemble des ligands ou localisé sur un seul ligand. Le problème de localisation/délocalisation de l'énergie d'excitation dans les complexes de coordination était encore récemment un sujet controversé, même pour $\left[\mathrm{Ru}(\mathrm{bpy})_{3}\right]^{2+}$ (bpy $=2,2^{\prime}$-bipyridine), le complexe de ruthénium (II) le plus étudié (Figure 1). Néanmoins un faisceau de résultats obtenus pour ce complexe par diverses techniques milite en faveur de la localisation électronique. En effet, la localisation de charge a été démontrée par spectroscopie par éclair laser nanoseconde pour un complexe bis-terpyridinique du ruthénium (II) comportant deux ligands équivalents [3]. Mais il a fallu avoir recours, plus récemment, à la spectroscopie d'absorption femtoseconde pour apporter la preuve de la localisation électronique dans le cas de $\left[\mathrm{Ru}(\mathrm{bpy})_{3}\right]^{2+}[4]$. La réaction peut donc s'écrire :

$$
\left.\left[\mathrm{Ru}^{\mathrm{I}}(\mathrm{bpy})_{3}\right]^{2+} \stackrel{\text { lumière }}{\longrightarrow}\left[\mathrm{Ru}^{\mathrm{MI}}(\mathrm{bpy})(\mathrm{bpy})^{*}\right)\right]^{2+*}
$$

Ceci signifie que la localisation électronique, qui est un acte élémentaire ultrarapide, constitue la première étape intramoléculaire du transfert d'électron et de la séparation des charges. Il est intéressant de noter qu'à l'état fondamental les ligands bpy sont équivalents et qu'ils ne le sont plus à l'état excité (réaction 1). Par conséquent, la localisation de charge implique une brisure de symétrie. Cette propriété se retrouve dans plusieurs processus biologiques, en particulier dans la photosynthèse naturelle. Il n'est donc pas étonnant que la photosynthèse artificielle qui tente de mimer les fonctions de la photosynthèse naturelle dans le but de convertir l'énergie solaire en énergie chimique, utilise de tels composés comme photosensibilisateurs dans les systèmes modèles de photolyse de l'eau en hydrogène ou oxygène [5], ou dans les piles photoélectrochimiques.

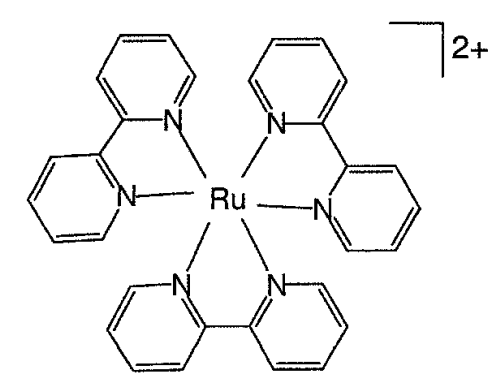

a)

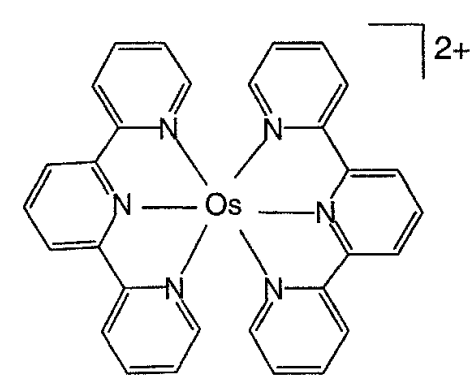

b)

Figure 1. Structure moléculaire des complexes: a) $[\mathrm{Ru}(\mathrm{bpy}) 3]^{2+}\left(\mathrm{bpy}=2,2^{\prime}\right.$-bipyridine), b) $[\mathrm{Os}(\mathrm{tpy}) 2]^{2+}\left(\right.$ tpy $=2,2^{\prime}: 6^{\prime}, 2^{\prime \prime}-$ terpyridine).

\section{TRANSFERT D'ELECTRON VECTORIEL}

Pour favoriser la localisation électronique, un des ligands bpy peut être modifié pour qu'y soit introduit un site accepteur d'électron de type phénazine pz éloigné du site métallique. C'est le cas du complexe 
$\left[\mathrm{Ru}(\mathrm{bpy})_{2} \mathrm{dppz}\right]^{2+}(\mathrm{dppz}=$ dipyrido-phénazine $)$ dont l'état fondamental comprend en effet deux entités distinctes identifiables, la première se comportant comme photosensibilisateur du type $\left[\mathrm{Ru}(\mathrm{bpy})_{3}\right]^{2+}$ et la seconde comme accepteur d'électrons du type phénazine [6]. L'excitation par la lumière conduit, ainsi que le montrent les spectres d'absorption de l'état excité obtenus par spectroscopie par éclair laser, à un transfert vectoriel de charges, dirigé de l'atome de Ru à la partie phénazine du ligand dppz. En d'autres termes, on observe un transfert intramoléculaire d'un électron et sa localisation sur le site accepteur d'un seul ligand. II en résulte une séparation de charges sur une distance d'environ $7 \AA$. Un tel complexe qui, sous l'action d'une perturbation lumineuse, est le siège d'une séparation vectorielle de charges, représente une étape vers la réalisation de photodiodes à l'échelle moléculaire. Outre son intérêt pour l'électronique moléculaire domaine qui se propose de traiter l'information par des molécules présentant des propriétés de composants électroniques - ce complexe de Ru qui présente deux sites redox, s'avère également intéressant pour la photosynthèse artificielle.

\section{TRANSFERT D'ELECTRON A LONGUE DISTANCE}

Afin de mieux comprendre dans quelles conditions le transfert d'électron et la séparation de charges s'effectuent lors des étapes primaires de la photosynthèse naturelle, les recherches se sont orientées vers la modélisation du site photosynthétique. L'élaboration de ces systèmes modèles à caractère biomimétique implique une démarche d'ingéniérie moléculaire. Une des idées consiste à construire un système supramoléculaire en associant un site photosensible PS et un site accepteur A (ou donneur D) d'électron. Dans une telle diade PS-A (ou PS-D), si les deux sites sont suffisamment éloignés l'un de l'autre, on peut espérer favoriser un transfert d'électron à longue distance et une séparation spatiale des charges.

Dans l'un des systèmes étudiés [7], le site accepteur (de type viologène $\mathrm{V}^{2+}$ ) est relié au site photosensible (de type pophyrine de zinc $\mathrm{ZnP}$ ) par une chaîne flexible ou semi-rigide. Des expériences de spectroscopie laser picoseconde dans des solvants de polarité différente suggèrent l'existence de plusieurs conformères. Le transfert d'électron intramoléculaire n'est pas observé pour la diade flexible. Il n'a lieu que pour l'un des conformères de la diade semi-rigide, notée $\left[\mathrm{ZnP}-\mathrm{Ph}-\mathrm{V}^{2+}\right]$. Ce conformère replié minimise la distance entre le site photosensible $\mathrm{ZnP}$ et le site accepteur d'électron $\mathrm{V}^{2+}$. Il en résulte une désactivation efficace de l'état excité de la porphyrine par le viologène, dans une réaction de transfert d'électron intramoléculaire conduisant à la formation d'une paire de charges à l'état excité (réaction 2) stabilisée par les solvants polaires.

$$
\left[\mathrm{ZnP}-\mathrm{Ph}-\mathrm{V}^{2+}\right] \stackrel{\text { lumière }}{\longrightarrow}\left[\mathrm{ZnP}^{*}-\mathrm{Ph}-\mathrm{V}^{2+}\right] \longrightarrow\left[\mathrm{ZnP}^{+\bullet}-{\mathrm{Ph}-\mathrm{V}^{+\bullet}}^{\mathrm{e}}\right]
$$

Cette étude confirme l'intérêt de concevoir des systèmes plutôt rigides et organisés, où l'on peut contrôler les distances entre les différents sites. Pour effectuer des transferts d'électron à plus longue distance et ainsi accroitre l'efficacité de la séparation de charges, des systèmes plus complexes ont été proposés [2,5]. Ils associent plusieurs accepteurs d'électrons $\mathrm{A}$ au site photosensible (comme par exemple la triade PS-A1-A2), ou des sites donneurs D séparés des sites A par le site photosensible (par exemple : triade D-PS-A, tétrade D-PS-A1-A2 ...). Dans de tels systèmes, l'électron photoexcité va passer rapidement d'un site à l'autre. Les charges électron-trou produites vont se trouver de plus en plus éloignées les unes des autres dans l'espace (réaction 3).

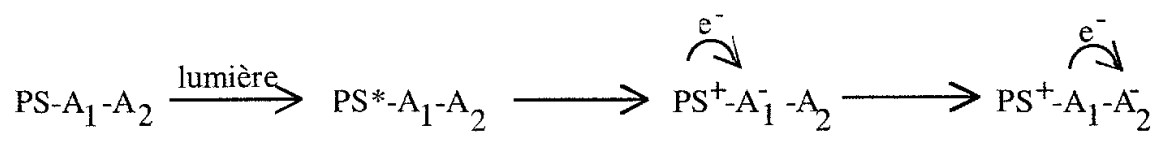


La possibilité d'une réaction de retour de l'électron, donc de la recombinaison des charges, est défavorisée. Ce qui accroît la durée de vie de l'état ultime de charges séparées (PS+ $-\mathrm{A}_{1}-\mathrm{A}_{2}^{-}, \mathrm{D}^{+}-\mathrm{PS}$ $\mathrm{A}^{-}$...) qui peut atteindre plusieurs microsecondes dans les systèmes les plus efficaces.

Ces systèmes constituent donc de bons modèles de la photosynthèse naturelle, car l'on sait, depuis l'élucidation en 1984 de la structure aux rayons $X$ du centre réactionnel d'une bactérie pourpre $[2,5]$, que celui-ci est un système moléculaire hautement organisé où tous les éléments sont ordonnés de manière bien précise avec des positions (distance, angle) parfaitement définies, et où l'absorption des photons provoque une brisure de symétrie, un flux d'électrons dans une direction bien déterminée sur de longues distances et une séparation spatiale des charges. En d'autres termes, les centres réactionnels photosynthétiques ainsi que les systèmes modèles du type polyade ont, comme le complexe $\left[\mathrm{Ru}(\mathrm{bpy})_{2} \mathrm{dppz}\right]^{2+}$, des comportements de photodiode moléculaire.

Dans cet esprit, nous avons élaboré récemment une nouvelle famille de polyades comportant un complexe terpyridine de ruthénium ou d'osmium (Figure 1) comme site photosensible, lié de façon covalente à un accepteur d'électron de type triarylpyridinium [8]. Ces systèmes, dont on peut ajuster les propriétés par substitution, présentent l'intérêt d'être rigides, de préserver la symétrie axiale du site terpyridinique, et d'être parfaitement définis avec une distance égale entre le métal et les sites donneurs D et accepteurs A d'électrons. Dans de tels systèmes, on peut espérer photoinduire un transfert d'électrons vectoriel sur une distance de $22 \AA$ et une séparation de charges de longue durée de vie. Outre leur intérêt pour l'électronique moléculaire (photodiode, fil) et la conversion photochimique de l'énergie solaire (séparation de charges), ces polyades possèdent des propriétés optiques non linéaires du troisième ordre très prometteuses (systèmes D-P-A conjugués) [9].

\section{CONCLUSION}

Au fur et à mesure des progrès réalisés dans la compréhension des processus de transfert d'électron, les chimistes et les physico-chimistes ont été conduits vers des structures moléculaires de plus en plus complexes et souvent de mieux en mieux organisées, et vers des applications multiples. Dans les quelques exemples présentés, les progrès sont certainement dus d'une part à ceux de la chimie de synthèse et d'autre part aux études des propriétés photophysiques et photochimiques de divers complexes de coordination qui servent d'éléments photoactifs dans les systèmes supramoléculaires étudiés. Ces études fondamentales qui constituent un passage obligé pour atteindre une meilleure compréhension des processus primaires en photochimie supramoléculaire, connaitront d'importants développements grâce aux techniques ultrarapides, notamment la spectroscopie laser femtoseconde. De telles études ouvrent ainsi la voie à la conception d'édifices structurés permettant d'optimiser et de contrôler le transfert d'électron intramoléculaire et in fine d'obtenir la séparation de charges, dans la perspective d'applications dans divers domaines: photosynthèse artificielle, électronique et photonique moléculaires, photobiologie, énergies renouvelables.

\section{Références}

[1] Lehn J.M., Angew. Chem. Int. Ed. Engl. 29 (1990) 1304-1319

[2] Balzani V., Scandola F., Supramolecular photochemistry (Ellis Horwood, New York, 1991)

[3] Amouyal E., Mouallem-Bahout M., Calzaferri G., J. Phys. Chem. 95 (1991) 7641-7649

[4] Damrauer N.H., Cerullo G., Yeh A., Boussie T. R., Shank C.V., McCusker J.K., Science 275 (1997) 54-57

[5] Amouyal E., Homogeneous Photocatalysis (J. Wiley, Chichester, 1997) pp. 263-307

[6] Amouyal E., Homsi A., Chambron J.C., Sauvage J.P., J. Chem. Soc. Dalton Trans. (1990) 1841 1845

[7] Shafirovich V. Y., Amouyal E., Delaire J., Chem. Phys. Letters 178 (1991) 24-30

[8] Lainé P., Bedioui F., Ochsenbein P., Marvaud V., Bonin M., Amouyal E., J. Am. Chem. Soc. 124 (2002) 1364-1377

[9] Konstantaki M., Koudoumas E., Couris S., Lainé P., Amouyal E., Leach S., J. Phys. Chem. B 105 (2001) 10797-10804 